\title{
Theoretical Analyses of Data Communications Integrated into Cordless Voice Channels
}

\author{
Radhakrishna, CANCHI. and Yoshihiko, AKAIWA. \\ Graduate School of Information Science \& Electrical Eng., \\ Kyushu University \\ 6-10-1, Hakozaki, Fukuoka 812-81, JAPAN \\ Phone: +81-92-642-3890 Fax: +81-92-632-5204. \\ E-mail : radha@mobcom01.is.kyushu-u.ac.jp
}

\begin{abstract}
Dynamic integration of data into voice channels of 2 nd generation cordless systems provides an effective channel utilization. This paper proposes and theoretically examines an Inhibit and Random Multi Access (IRMA) protocol for data terminals in the integrated voice and data system. Analytical expressions are derived to quantify the effect of data inhibition on its performances, i.e. throughput and delay for both infinite and finite population model. We investigate the data performance in two extreme situations: 1 . No voice load and 2 . Full voice load. The numerical results indicate that a comparable performance can be achieved.
\end{abstract}

TDMA, IRMA, Throughput, Delay

\section{Keywords}

\section{INTRODUCTION}

The last decade has witnessed an unprecedented development in the arena of wireless data communication systems (Kaveh Pahlavan,1994). The advent of digital cellular and cordless systems primarily for voice applications and local and wide area data networks for data oriented services, has stimulated the ideas and efforts for integration of services in order to evolve Personal Communication Networks. At the other end, the advances in computer technology and signal processing, spurred by the miniaturization, have shown the feasibility of mobile computing as well as personal voice and data communication using a portable device.

Various schemes have been proposed in literature for voice and data integration in wireless networks ((Akaiwa,1994), (GangWu,1994), (Jeffrey,1995), (Sanjiv,1994)). This paper examines an Inhibit and Random Multi Access (IRMA) protocol (Akaiwa,1994) for data transfers to be integrated into voice oriented digital channels of cordless systems such as PHS during the silence periods of speech activity. The data transmission performance degrades due to the constraint, i.e. data inhibition, provided in the integrated voice and data system. We 
derived analytical expressions to quantify the effect of data inhibition on the performances, i.e., throughput and delay, of the data communications. In our analyses, we consider both infinite and finite number of data terminals. Finally, a finite population case of integration is modeled and analyzed by assuming an approximate Markovian process. The expressions for average throughput and average delay are obtained for a simple two state speech model.

\section{SYSTEM DESCRIPTION}

The integrated system consists of a base station and a number of terminals, separate for voice and data, that share a common radio channel with a pair of frequencies. The basic access scheme assumed for voice terminals is TDMA with an assignment of channel on demand and an IRMA for data terminals. The base stations broadcast in the format of frames of fixed length that is further divided into equal time slots. The length of each slot is identical to that of a packet. Each packet is an integral of actual information bits and some overheads required for synchronization and signaling. Our area of investigation is limited to the communication between cordless terminals and their base stations.

\section{IRMA PROTOCOL FOR DATA COMMUNICATIONS}

The data communications are controlled by the respective base stations by broadcasting appropriate control signals at the beginning of each slot. By assuming a voice activated transmission, we dynamically integrate the data into the channel during silence periods of speech activity. Whenever a silence period is detected in a channel occupied with voice communications, the base would indicate this event by broadcasting the corresponding signal in the down link. The data terminal transmits in the form of bursts of duration equal to one time slot. Since the system is voice oriented, the crucial issue is how to avoid collision between voice and data packets. The first packet of the resumed talkspurt indicates the restart of voice activity with a view to avoiding any loss of voice packets. In order to resolve any collisions between voice and data whenever speech transmission is resumed, data terminals are inhibited from transmissions in the following slot and then allowed to access the channel randomly. In other words data terminals follow an IRMA protocol for channel access.

\section{ANALYSES}

\subsection{Case 1: Infinite data terminals and no voice load}

The system is assumed to have infinite data terminals and the propagation delays over radio channel is quite small compared to the packet transmission time. Each user have almost a packet ready for transmission. We assume no voice traffic, i.e., all slots are available for data transfers. However, data transmission is inhibited after every collision. The average offered load, G, follows the Poisson distribution. The activity on the channel can be divided into cycles where each cycle contains zero or one or more idle events, an active event and an inhibit event in case of a collision and busy events during reserved slots. The throughput of the channel, $S$, is the average successful duration in a given cycle of operation. The normalized throughput without any reserved slots is derived as

$$
S=\frac{G e^{-G}}{2-e^{-G}-G e^{-G}}
$$


This is compared with the throughput of Slotted ALOHA $S=G e^{-G}$ as in the Figure 1. The maximum throughput achievable is less than that of S-ALOHA and is because a slot is unutilized following every conflict. The throughput obtained for the infinite model reaches a maximum value of $30.2 \%$ at an offered load of $76.8 \%$. This throughput value is comparable to that of Slotted-ALOHA where the maximum throughput is $36.8 \%$ at an offered load of unity. Moreover, at lighter loads, the effect of inhibition on data transfers is insignificant and throughput is identical to that of S-ALOHA. Furthermore, in typical ALOHA networks the channel traffic is usually in the range of 0.10 or less(Norman Abramson,1994), in which case our system offers the effective usage of the channel.

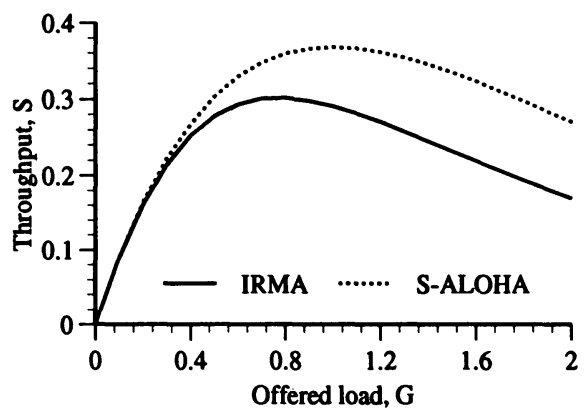

Figure 1 Throughput characteristics of infinite data terminals.

The delay analysis accounts newly arrived and retransmitted packets as separate variables(Kleinrock,1984). The retransmissions take place randomly in one of the following $k$ slots $(1 \leq k \leq K)$ after a collision is confirmed and each slot being equally likely with probability $1 / K$, where $K$ is a backoff parameter. The more accurate expressions of normalized throughput $S$ and delay $D_{n}$, by considering the delay experienced by collided packets, are obtained as

$S=G \frac{q_{r}}{q_{r}+1-q_{n}}$

where

$$
\begin{aligned}
& q_{n}=\left[\frac{G}{K} e^{-G}+e^{-\frac{G}{K}}\right]^{K}\left(\frac{1}{2-e^{-G}-G e^{-G}}\right) e^{-S}, \\
& q_{r}=\left[\frac{e^{-\frac{G}{K}}-e^{-G}}{1-e^{-G}}\right]\left[\frac{G}{K} e^{-G}+e^{-\frac{G}{K}}\right]^{K-1}\left[\frac{1}{2-e^{-G}-G e^{-G}}\right] e^{-S} \text { and } \\
& D_{n}=1+H\left(1+\frac{K+1}{2}\right)
\end{aligned}
$$

where $H=(G / S-1)=$ average retransmissions given in terms of $S, G$ and $K$. 
Figure 2 depicts the delay performance given by the equations (2) and (3) for the infinite terminals model. The delay that a data packet experiences, in terms of slots, is obviously higher than that of S-ALOHA since a slot is unutilized by data terminals for every collision. Nevertheless, the delay values are comparable to that of S-ALOHA.

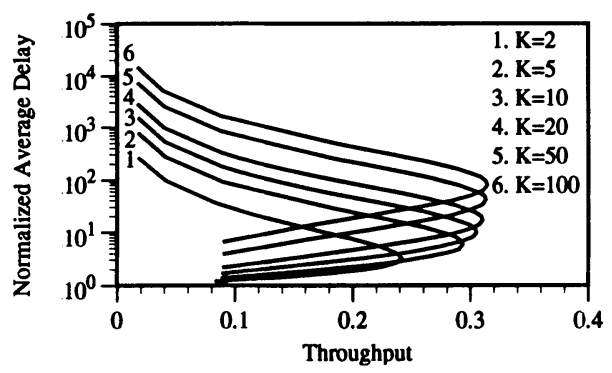

Figure 2 Delay characteristics of infinite data terminals.

\subsection{Case 2: Finite data terminals and heavy voice load}

A finite population case of integration is modeled and analyzed by assuming an approximate Markovian process. The expressions for average throughput and average delay are obtained for a simple two state speech model. In this section, we derive the expressions for throughput and delay with the help of Steady (Equilibrium) State Analysis .

Since the system is voice oriented, the blocking probability of the integrated system is same as that of voice only system. In this section we donot consider the signaling traffic of voice users and call set up procedures but we assume fixed number of connections by voice users all the time. Though this fixed number of connections can be evaluated by considering some performance measures, such as blocking probability, we assume a typical heavy (full) loading conditions during busy hour where all channels (slots) are occupied voice. In other words, the fixed number of connections equal to the number of slots in a TDMA frame. Let each TDMA frame be divided into $N$ equal time slots, of which $N_{v}$ slots, where $0 \leq N_{v} \leq N$, are always occupied by voice calls. In the present case $N_{v}=N$.

\section{Voice Traffic Modeling}

For voice traffic, the talkspurts and silence periods within a voice call are also exponentially distributed with means $T_{t}=1 / \alpha$ seconds and $T_{s}=1 / \beta$ seconds, respectively. The steady state (equilibrium) for Talkspurt and Silence can be obtained as

$$
P_{T}=\frac{P_{S 2 T}}{P_{T 2 S}+P_{S 2 T}} \text { and } P_{S}=\frac{P_{T 2 S}}{P_{T 2 S}+P_{S 2 T}}
$$

where $P_{T 2 S}$ and $P_{S 2 T}$ are Talkspurt to Silence and Silence to Talkspurt transition probabilities in a slot duration $T \mathrm{sec}$, respectively.

\section{Data Traffic Modeling}

The data traffic is assumed to be an approximate Poisson process with arrival rates $\lambda_{d}$ messages per second. The data message length is assumed to have a geometric distribution with mean $1 / \delta_{\mathrm{d}}$. Consider a finite number of data terminals $M$. The behavior of each terminal can be described by a model with three states : data generating(DG), contending for a channel (DC), and transmitting data packets (DT) successfully. 
By assuming the discrete random process points at the beginning of frames, consider a point of time $t$ at the beginning of a frame " $x$ " $(x$ is an integer) as shown in Figure 3. If $l x, m x, k x$ denotes the number of data terminals generating, contending and transmitting respectively, then the frame $x$ can be described by state vector $\{l x, m x, k x\}$. Since the total number of terminals is finite and constant, $M$, the state vector can be simplified to $\{m x, k x\}$ where $l x$ is given as $\{M$ $m x-k x\}$. Since the transition to the state $\left\{m_{x+1}, k_{x+1}\right\}$, at $t+1$, depends only on the events at $t$, we can follow a Markovian analysis. A steady state distribution exists for this process since $0 \leqq m_{x} \leqq M$ and $0 \leqq k_{x} \leqq N$

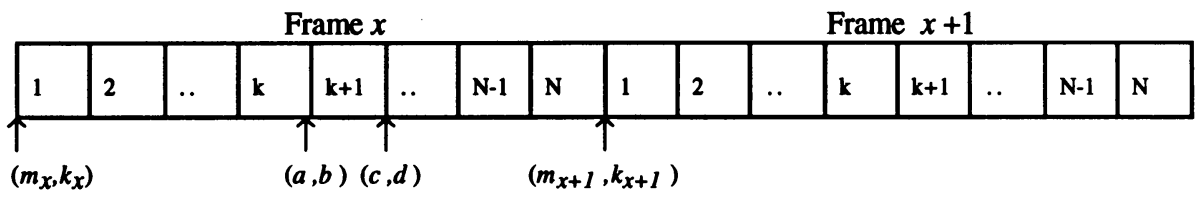

Figure 3 TDMA frame and instants of state transitions.

If $\pi_{i j}$ denotes the steady state probability of the system in state $(i, j)$ and $p_{i j r s}$ denotes the one step state transition probability from state $(i, j)$ to $(r, s)$, the steady state probabilities given by solving a set of linear equations

$\Pi=\Pi P$ and $\Sigma \pi_{i j}=1$,

where $\Pi=\left\{\pi_{i j}\right\}=$ steady state vector, and $\boldsymbol{P}=\left\{p_{i j r s}\right\}=$ State Transition Matrix.

Since each frame has $N$ slots and the state at the end of the $N$ th slot gives the state at $t+1$, i.e., at the beginning of frame $x+1$. It is enough, if we evaluate the state at the end of $N$ th slot for a given state at $t$. To arrive at this, we consider all possible state transitions within the frame of $x$ by adopting an approximate Markovian analysis again.

While $(a, b)$ being the state vector at the end of $k$ th slot, $k+1$ th time slot (channel) of frame $x$ exists in one of the following states as far as the data terminals are considered :

1. Talkspurt state

2. Occupied by a data terminal

3. Inhibit state

and

4. Idle state

Let $P_{T S}, P_{D T}, P_{I N}$ and $P_{I D L E}$ denotes the probabilities of a channel being in the above states, respectively and can be obtained as

$$
\begin{aligned}
& P_{T S}=P_{T} \frac{N_{v}}{N}=\frac{P_{S 2 T}}{P_{T 2 S}+P_{S 2 T}} \frac{N_{v}}{N} \\
& P_{D T}=\left(1-P_{T S}\right) \frac{b}{N} \\
& P_{I N}=1-P_{T S}-\left(1-P_{T S}\right)(1-p)^{a}+\left(\left(2 P_{T S}\right)-1\right) P_{S T}
\end{aligned}
$$

Then the probability of an idle state is given by

$$
P_{I D L E}=1-P_{T S}-P_{D T}-P_{I N}
$$


The probabilty that a voice slot is in silence state is given as $P_{S I L}=1-P_{T S}$. The elements of $\boldsymbol{P}$ are obtained by an iterative equation given as

$p_{b}(c, d)=\sum_{b=0}^{N} \sum_{a=0}^{M-b} q_{a b c d} p_{i j}(a, b)$

where $a=m_{x}(k)=m_{x} @$ the end of $k$ th slot.

$b=k x(k)=k x @$ the end of $k$ th slot.

$c=m x(k)=m_{x} @$ the end of $k+1$ th slot.

$d=k_{x}(k)=k_{x} @$ the end of $k+1$ th slot.

$p_{i j}(a, b)=$ The probability of state $\{a, b\}$ given the state at the start of frame, i.e. $(i, j)$.

$q_{a b c d}=$ one step(slot) transition probability from state $(a, b)$ to $(c, d)$ taking into

account of the probabilities of silence state, inhibit state of the channel etc.

The matrix $\boldsymbol{P}$ is constructed by using the iterative equation (10) for all possible values of $i, j$, where $0 \leqq i \leqq M-i$ and $0 \leqq j \leqq N$ and then steady state vector $\left\{\pi_{i j}\right\}$ is calculated.

The total average data throughput for the finite population model during the silence periods of channel is given by

$S=S_{r}+S_{c}$

where $S_{r}$, the ratio of the average number of data packets successfully transmitted in a frame to the number of slots $(N)$ in a frame by taking average silence and inhibit probabilities, $P_{S I L}$ and $P_{I N}$ into account, and $S_{c}$, the average number of data terminal that are successful in a slot in an equilibrium state given a data generation rate $\sigma$, can be evaluated as

$S_{r}=\frac{P_{S I L}}{N} \sum_{k=0}^{N} \sum_{m=0}^{M-k} k \pi_{m k}$ and $S_{c}=M \sigma-\sum_{k=0}^{N} \sum_{m=0}^{M-k}(m+k) \pi_{m k} \sigma$

The total average delay experienced by data packets for this case is given as

$\mathrm{D}=D_{c}+D_{r}($ Packet Length $)=D_{c}+\operatorname{Dr}\left(\left(1 / \delta_{d}\right)-1\right)$

where, By Little's results,

$D_{c}=\frac{\sum_{k=0}^{N} \sum_{m=0}^{M-k} m \pi_{m k}}{S_{c}}$ and $D_{r}=\frac{\sum_{k=0}^{N} \sum_{m=0}^{M-k} k \pi_{m k}}{S_{r}}$

\section{NUMERICAL RESULTS OF THE PERFORMANCES}

In order to obtain the numerical solutions, the iterative procedure of relaxation is applied to the equations (5), i.e.,

$\Pi=\lim _{n \rightarrow \infty} \Pi_{0} P^{n}$ 
where $\boldsymbol{n}$ is chosen sufficiently large so that truncation error is negligible and the calculation is terminated when iterative values of $\Pi$ converges. For numerical results the system with the parameters listed in Table 1 is considered.

Table 1 System Parameters

\begin{tabular}{lll}
\hline Parameter & Symbol & Nominal value \\
\hline TDMA frame length (m.sec) & $\mathrm{T}$ & 5 \\
Slots per TDMA frame & $\mathrm{N}$ & 4 \\
Average talkspurt duration $(\mathrm{sec})$ & $\mathrm{Tt}$ & 1 \\
Average silence duration $(\mathrm{sec})$ & $\mathrm{Ts}$ & 1.35 \\
Voice source coding $(\mathrm{Kbps})$ & $\mathrm{Rs}$ & 32 \\
Data terminals & $\mathrm{M}$ & $25 \quad$ (variable) \\
Data generation rate ( packets /slot $)$ & $\sigma$ & 0.004 \\
Mean data length rate & $\delta \mathrm{d}$ & $0.1 \quad$ (variable) \\
Probability of data transmission & $\mathrm{p}$ & $0.1 \quad$ \\
\hline
\end{tabular}

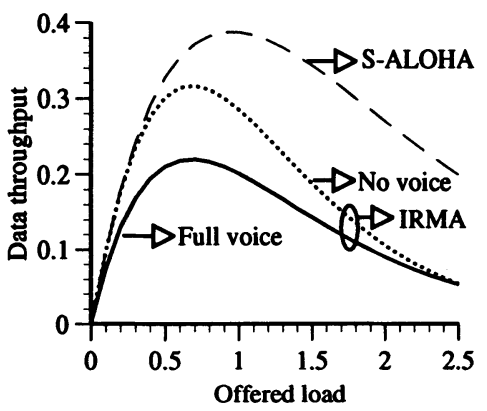

(a) Without reservation

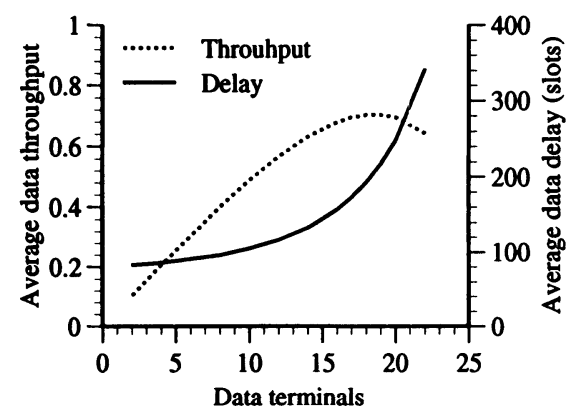

(b) With reservation

Figure 4 Performance characteristics of finite population model.

The performance achieved with the IRMA protocol with the transmit probability 0.1 is shown in Figure 4. Figure 4(a) shows the throughput curves for No voice load and Full voice load for the finite data terminals without any slot reservation facility. The maximum throughput per silence duration during heavy voice load conditions found to be $22 \%$. Figure 4(b) represents the average throughput and delay performances as the number of terminals with slot reservation facility per base station is varied. The maximum number of data terminals per base station for stable operation is 19 and the corresponding throughput and delay are $70.44 \%$ and 216.79 slots respectively. Figure 5 shows the performance for $\mathbf{1 6}$ data terminals as the transmission probability is varied. The optimum range of transmit probability over which high throughput and low delay can be achieved is found to be $0.05-0.15$.

\section{CONCLUSIONS}

An IRMA (Inhibition based Random Multiple Access) protocol suitable for data integration into the voice channels of digital cordless system has been theoretically analyzed. The analytical results indicate that a comparable performance of data communications can be achieved by integrating data during the silence duration of speech activity despite the constraint,i.e. inhibition, on the random access of data terminals . 


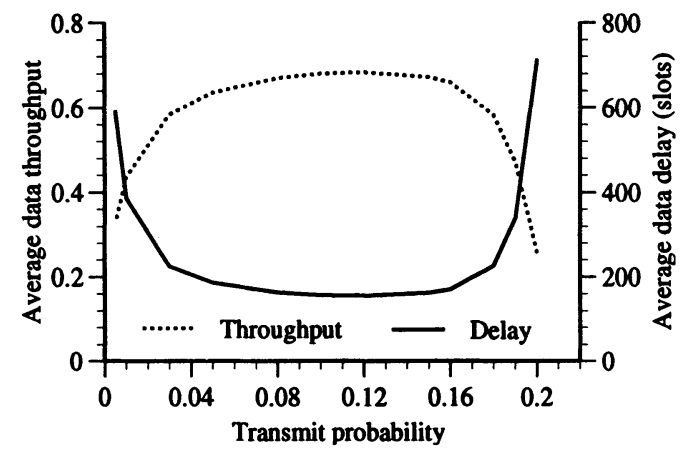

Figure 5 Performance curves versus transmit probability with $M=16$.

\section{REFERENCES}

Akaiwa, Y. and Nakajima, A.(1994), Integration of wireless local area data communication to digital cordless telephone channel, Proceedings of IEEE International Conference on Personal Wireless Communications, 190-3.

Gang Wu, K. Mukumoto and A. Fukuda (1994), Integration of Voice and Data in Wireless Information Networks with Data Steal into Voice Multiple Access, IEICE Transactions on Communications, Vol.E77-B, 939-47.

Jeffrey E. Wieselthier and Anthony Ephremides(1995), Fixed and Movable-Boundary ChannelAccess Schemes for Integrated Voice/Data Wireless Networks, IEEE Transactions on Communications, VOL.43, 64-74

Kleinrock, L. (1976) Queuing Systems, Vol.2, Computer Applications, New York:

Kaveh Pahlavan et al.(1994) Wireless Data Communications, Proceedings of the IEEE, Vol. 82, 1398-430.

Sanjiv Nanda (1994), Stability Evaluation and Design of the PRMA Joint Voice Data Syatem, IEEE Transactions on Communications, VOL.42, 2094-104:

Norman Abramson, Multiple Access in Wireless Digital Networks, Proceedings of the IEEE, Vol. 82, 1360-70.

\section{BIOGRAPHIES}

RADHAKRISHNA CANCHI received an M.Tech degree in electronics engineering from Regional Engineering College, Warangal, India. He has been a research engineer in C-DOT, India since 1989. He has been involved in the development of TDMA Point to Multi Point radio communications at C-DOT. He was awarded Hindu-Hitachi Scholarship and undergone a training at Hitachi Telecommunication Division, Yokohama for six months in 1992. Now, he is a student of doctorate course in Intelligent Systems at Kyushu University on MONBUSHO (Japanese Government ) 1994 Scholarships. He is a student member of IEEE.

YOSHIHIKO AKAIWA received his doctorate degree in electronics engineering from Kyushu University in 1979. He joined Central Research Laboratories, NEC in 1968. Later he moved to Kyushu Institute of Technology where he was a professor from 1988 to 1996 . Now he is a professor at Kyushu University. He has been involved in the research and development of mobile radio communication techniques for 20 years. He received the paper award and the distinguished achievement award from IEICE, Japan and the Avant Garde award from IEEE Vehicular Technology Society. He is a member of IEICE, Japan and a senior member of IEEE. 